\title{
Laparoscopic Triple Segmental Bowel Resection for Endometriosis Revealed by Rectal Obstruction during Infertility Treatment
}

\author{
Kunihiko Nagakaria ${ }^{a}$ Daisuke Azuma ${ }^{a}$ Kazuhiro Takehara ${ }^{a}$ \\ Masakazu Ohuchi ${ }^{a}$ Yoichi Ishizaki ${ }^{a}$ Kazuhiro Sakamoto ${ }^{b}$ \\ aDepartment of Surgery, Juntendo University Urayasu Hospital, Urayasu, Japan; \\ ${ }^{b}$ Department of Coloproctological Surgery, Juntendo University Faculty of Medicine, Tokyo, \\ Japan
}

Keywords

Bowel obstruction · Bridge to infertility treatment · Endometriosis · Laparoscopy

\begin{abstract}
Intestinal endometriosis is a benign disease characterized by ectopic growth of the endometrium and causes extensive fibrosis and adhesions in response to repeated episodes of bleeding and inflammation with the menstrual cycle. We encountered a rare case of intestinal endometriosis that caused complete rectal obstruction in a 34-year-old woman undergoing infertility treatment. Colonoscopy showed rectal stenosis and obstruction but no evidence of a tumor. Bowel obstruction due to endometriosis was diagnosed based on the history and imaging findings. Transanal decompression was performed. Subsequent laparoscopic surgery revealed severe inflammation around both ovaries and a tumor-like rectal stenosis. Similar findings were obtained in the transverse colon and terminal ileum. We performed laparoscopic low anterior resection, partial transverse colon resection, ileocecal resection, bilateral cystectomy, and left salpingectomy. Infertility treatment was restarted and resulted in a successful term pregnancy. The patient remains well. Laparoscopic surgery, which has the advantage of being minimally invasive, allows for early postoperative recovery and discharge in patients with endometriosis; furthermore, the uterus and adnexa can be preserved due to the magnifying effect of the laparoscope. In this case, it was possible to resume infertility treatment. Intestinal endometriosis is a rare cause of bowel obstruction, but should be kept in mind if intestinal obstruction occurs during infertility treatment. Laparoscopic surgery may be useful for multiple endometriotic lesions and serve as a bridge to infertility treatment.
\end{abstract}


Nagakari et al.: Laparoscopic Bowel Resection for Endometriosis during Infertility Treatment

\section{Introduction}

Endometriosis is a benign disease caused by ectopic growth of the endometrium. Histological findings include fibrotic endometrial tissue and smooth muscle metaplasia from the muscularis propria to the subserosa, with extensive fibrosis and adhesions as a result of repeated cycles of hemorrhage and inflammation during the menstrual cycle. When severe, endometriosis may cause melena and intestinal obstruction. Here, we describe a case of rectosigmoid obstruction during infertility treatment in a woman with endometriosis that was treated successfully by laparoscopic triple segmental bowel resection. Intestinal endometriosis in three separate locations is rare, and a favorable course was achieved by laparoscopic surgery.

\section{Case Presentation}

A 34-year-old woman who was undergoing infertility treatment presented to our hospital with a 1-month history of constipation and abdominal fullness. She complained of abdominal pain, vomiting, and mild fever and reported not passing gas for several days. She mentioned a history of surgery for endometriosis at 22 and 30 years of age and 5 previous cycles of infertility treatment. She had no history of rectal bleeding.

On examination, she looked unwell and complained of acute lower abdominal pain. Her vital signs were normal except for a blood pressure of 147/85 mm Hg. The abdomen was distended and tympanic with hyperactive bowel sounds. No palpable masses were found. Digital rectal examination revealed an empty rectum. Laboratory investigations were normal, except for a CA-125 level of 344.6 U/mL. Abdominal radiographs showed an extensively dilated and fluid-filled small intestine and colon. Abdominal computed tomography scans showed a circumferentially thickened rectal wall and dilatation of the proximal colon. The border between the rectum and the uterus was unclear. Complete bowel obstruction was confirmed (Fig. 1a, b). Pelvic magnetic resonance imaging findings were similar to the computed tomography findings. Colonoscopy showed a constricted area $11 \mathrm{~cm}$ distal to the anus but no tumor. A gastrografin enema revealed a 4-cm stenosis in the rectum. A guide wire was passed through the stenosis to confirm continuity of the gastrointestinal tract (Fig. 1c).

No menstruation-related symptoms were noted. However, endometriosis was suspected based on the history and examination findings. Urgent laparoscopic surgery was performed, which revealed a thickened and edematous bowel wall, bloody cyst fluid in the anterior rectal wall near the site of the endometriotic lesion, adhesions between the rectum and uterus, and peritoneal endometriosis involving the pouch of Douglas (Fig. 2a). In addition, similar fibrosis and strictures were found at the terminal ileum and transverse colon, suggesting a diagnosis of intestinal endometriosis (Fig. 2b, c). After the adhesions of the sigmoid colon were detached from the outside, the ureteral and gonadal vessels were preserved by a medial approach. Next, the superior rectal artery and sigmoid artery were dissected. The ureter was identified cephalad. Detachment was started from the posterior of the rectum, which was relatively easy to dissect. The lateral side was dissected from the cranial end of the ureter toward the adhesion. The anterior wall was dissected using the uterine wall as a guide. Fibrosis was severe, and dissection was difficult. When caudal detachment was advanced from the peritoneal reflection, we confirmed that there was no endometriosis in the intestinal tract (Fig. 2d). An adequate margin was secured, and the dissection was performed using an ultrasonic device (iDrive $^{\mathrm{TM}}$; Covidien, Mansfield, MA, USA). There was no damage to the hypogastric nerve, pelvic plexus, or ureter, and no postoperative dysuria was observed. The right colon was sufficiently separated at the distal ileum and mobilized to perform ileocecal resection, and the transverse colon was mobilized as far as possible. At this point, the umbilical port was

\section{Karger'}




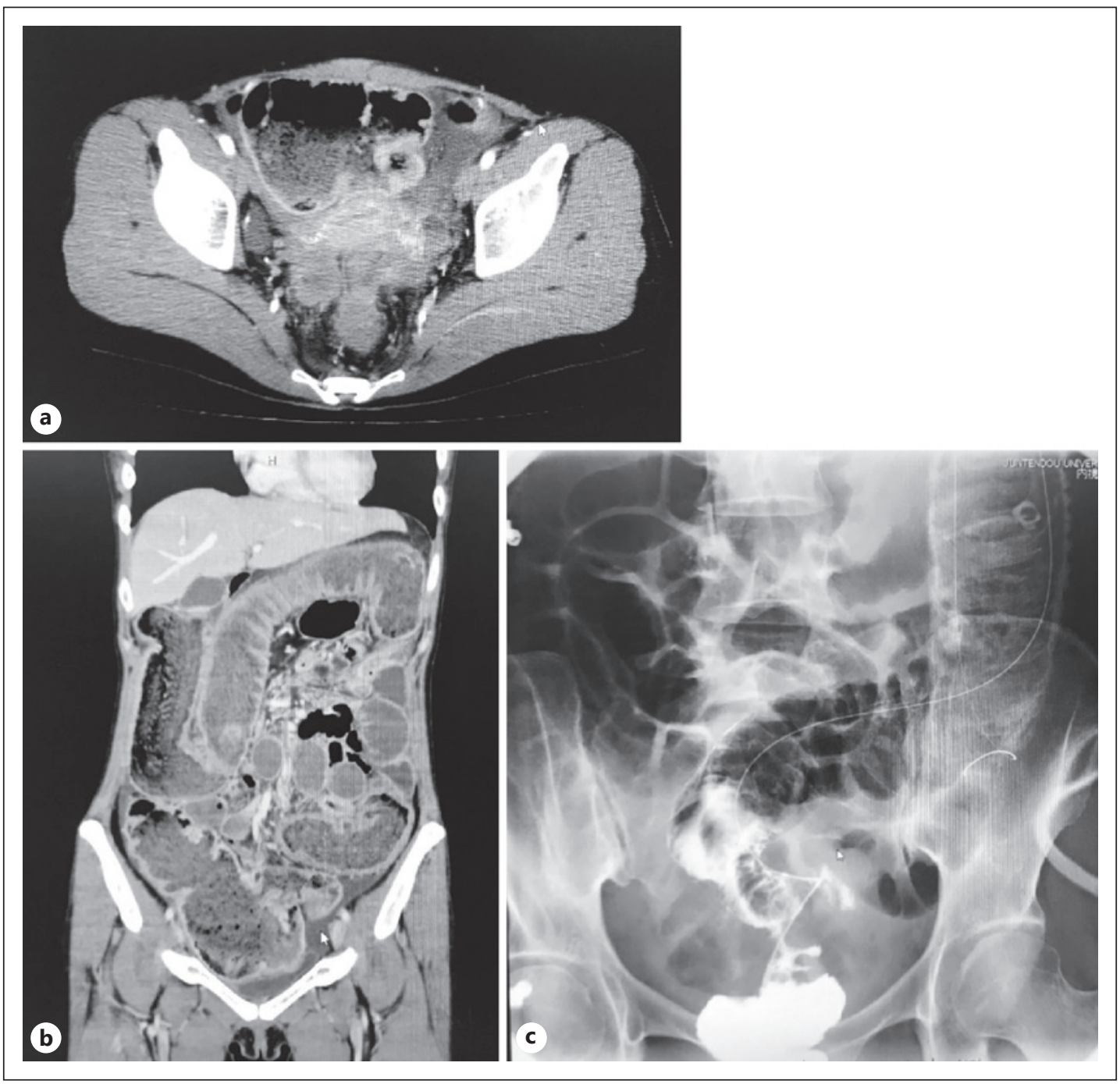

Fig. 1. Preoperative findings. a, b Abdominal enhanced computed tomography scan showing extensive dilation of the intestine. The border between the rectum and the uterus is unclear. c Gastrografin enema showed a 4 -cm stenosis in the rectum.

expanded to $5 \mathrm{~cm}$ to become extracorporeal, and the entire small bowel was inspected manually via minilaparotomy. The affected segments were limited to the rectum, transverse colon, and terminal ileum. The rectum was sutured laparoscopically using a double-stapling technique (Fig. 2e), and functional end-to-end anastomosis of the ileum and transverse colon was performed after the mesentery was processed outside the body. The endometriosis appeared to have directly invaded the rectum and developed ectopically in the ileum and transverse colon. A residual cyst was subsequently found in the left fallopian tube, and bilateral ovarian cystectomy + left salpingectomy + right para-ovarian cystectomy were performed. A drain was inserted at the anastomosis site, and the operation was completed (Fig. 2f). The total operating time was $302 \mathrm{~min}$, and the estimated blood loss was $40 \mathrm{~mL}$.

\section{Macroscopic Findings in Resected Specimens}

No epithelial changes were observed in the rectum, transverse colon, or ileum. The intestine and mesentery were taut and narrowed due to severe inflammation (Fig. 3a, b). 
Nagakari et al.: Laparoscopic Bowel Resection for Endometriosis during Infertility Treatment
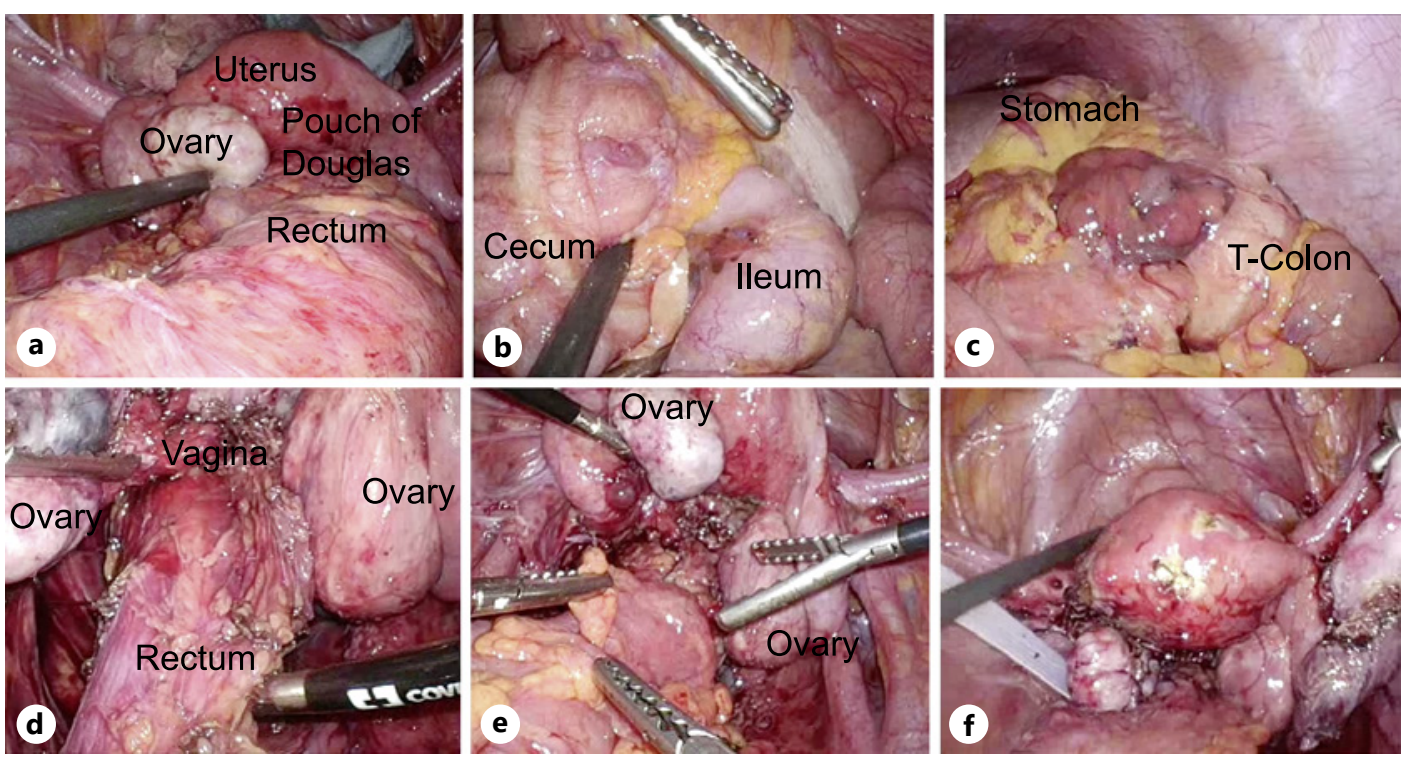

Fig. 2. Operative findings (laparoscopic view). The wall of the colon was thickened and edematous. There was bloody cyst fluid in the anterior rectal wall near the endometriotic lesion. There were adhesions involving the rectum and uterus. a Laparoscopic observation revealed peritoneal endometriosis in the pouch of Douglas. b, c Fibrosis was observed in the terminal ileum and transverse colon (T-Colon) with wall thickening and stricture. The diagnosis was intestinal endometriosis. $\mathbf{d}$ Exposure of the pouch of Douglas after detachment from the rectum. e After double-stapling. $\mathbf{f}$ A drain was inserted at the anastomosis site, and the operation was completed.

\section{Histopathological Findings}

Endometriosis consisting of endometrial gland ducts and interstitium was found from the muscularis propria to the subserosal layer in the rectum, ileum, and transverse colon. There were no findings suggestive of malignancy (Fig. 3c, d).

\section{Postoperative Course}

The postoperative course was uneventful, and the patient was discharged on hospital day 16. She then resumed infertility treatment. One month later, CA-125 decreased to $63 \mathrm{U} / \mathrm{mL}$, and pregnancy was confirmed 3 months later. She gave birth safely at 37 weeks 6 days of gestation.

\section{Discussion}

Endometriosis is a benign disease in which endometrial tissue grows ectopically. Intestinal endometriosis is reported to occur in $3-34 \%$ of all cases $[1,2]$. The most frequent location of bowel involvement with endometriosis is the sigmoid colon (over $65 \%$ of cases), followed by the rectum, the ileum, the appendix, and the cecum [3]. The most widely accepted concept regarding the etiology of peritoneal endometriosis is Sampson's theory of transtubal transplantation. However, alternative theories include direct transplantation, whereby a piece of endometrial tissue is transplanted by surgery or delivery and fetal epithelium derived from the remnants of the Mullerian ducts and Wolffian ducts. However, consensus remains elusive [4]. In our case, in addition to the main lesion, multiple lesions were found in the pouch of Douglas and the gastrointestinal tract. Furthermore, there was a history of surgery for endometriosis, suggesting the possibility of both transtubal and direct transplantation. 
Nagakari et al.: Laparoscopic Bowel Resection for Endometriosis during Infertility Treatment



Fig. 3. Pathological findings. a, b Macroscopic findings in the resected rectal specimen. The cross-sectional image of the resected specimen showed fibrosis from proper muscle to subserosal layer. There were brown spots in the layer of proper muscle (b*). c, d Microscopic findings in the resected rectal specimen (hematoxylineosin staining). An endometorial tissue was showed in muscular layer and subserosal layer (arrow).

The occurrence of endometriosis has increased in recent years, as has the number of reported cases. The clinical manifestations are abdominal pain, melena, dysesthesia, and difficulty in defecation, which appear to fluctuate in severity according to the menstrual cycle $[5,6]$. Intestinal obstruction can occur when an intestinal stricture becomes severe.

The diagnosis of endometriosis is based on history and clinical signs, but there is no specific diagnostic test, and several tests must be combined. Magnetic resonance imaging is reported to be particularly useful because endometriosis shows high signal intensity on T1-weighted images and low signal intensity on T2-weighted images [7]. On endoscopy, endometriosis often appears as a submucosal lesion; however, the positive biopsy rate is low because the main site of the lesion extends from the subserosa to the muscularis propria, and the preoperative pathological diagnosis rate is less than 10\% [8]. In most cases, postoperative pathological examination provides a definitive diagnosis.

In a reported case, CA-125 was high, but preoperative biopsy was not diagnostic. It should be noted that cancer develops in $0.3-1 \%$ of patients with endometriosis [9]. The differential diagnosis includes primary malignant tumor, submucosal tumor, metastatic tumor, and inflammatory disease, such as Crohn's disease. Careful differentiation is required because of the different treatment methods. Indications for surgery include cases in which malignant tumors cannot be ruled out, intestinal obstruction that cannot be improved by conservative 
Nagakari et al.: Laparoscopic Bowel Resection for Endometriosis during Infertility Treatment

treatment due to severe fibrosis, hormone therapy resistance, and desire to become pregnant when hormone therapy cannot be applied. Patients with endometriosis who require infertility treatment are thus candidates for surgery. Moreover, surgical treatment is indicated in cases where endometriosis causes bowel obstruction, as in our patient.

Minelli et al. [10] reported a recurrence rate of $8.4 \%$ in patients who underwent resection of intestinal endometriosis, which suggests that drug therapy may be needed to prevent postoperative recurrence. Furthermore, $12.3 \%$ of their patients had intraoperative and postoperative complications, and additional surgery was required in $80 \%$ of these cases [10]. Therefore, the indications for surgery should be carefully considered, and patients should be given sufficient explanation before consenting to surgery. Our patient had no intraoperative or postoperative complications, and her postoperative course was favorable. No hormonal therapy was administered, and she was able to resume infertility treatment.

Surgery is difficult when intestinal endometriosis is deep and involves the rectum. The anatomical characteristics of the densely packed organs within the limited space of the pelvic cavity are difficult to appreciate, and the normal anatomy of the deep pelvis is distorted when the pouch of Douglas is obstructed by inflammation or adhesions. The risks of ureteral injury, pelvic nerve injury, and intestinal perforation are high during surgery. The key steps in the surgical procedure were starting the detachment from the less-inflamed cranial side, identifying the ureter, and then proceeding to detachment to the caudal side of the pouch of Douglas, with the uterus and vaginal wall as a guide. Potential postoperative problems include urination disorders and suture failure at the anastomosis site. However, laparoscopic surgery is useful when working within the limited space of the pelvic cavity, and the magnifying effect allows anatomical structures to be recognized more easily. Thus, detachment can be performed more safely, and the surrounding organs can be preserved more reliably.

In our case, laparoscopic surgery was selected for cosmetic reasons and to allow for immediate laparotomy depending on the findings. With the widespread use of laparoscopic surgery in recent years, an increasing number of cases of intestinal endometriosis are being diagnosed intraoperatively. Ruffo et al. [11] reported a series of 31 cases of endometriosis in the ileocecal region that was found incidentally in all cases because it was associated with endometriosis at other sites.

Laparoscopic surgery for intestinal endometriosis is becoming increasingly common. Recently, there have been reports of single-port surgery, mostly performed for endometriosis involving the ileum, sigmoid colon, or rectum alone. To our knowledge, only one other case has been reported that involved simultaneous surgery on the rectum, ileum, and colon [12].

Laparoscopic surgery has been shown to have a higher fertility preservation rate than open surgery in patients with intestinal endometriosis [13]. Furthermore, it results in fewer postoperative adhesions than open surgery, which suggests that laparoscopic surgery may have less of an impact on functioning of the fallopian tubes and ovaries [14].

Laparoscopic surgery is useful in terms of being minimally invasive and also has the additional advantages of allowing detailed observation of the abdominal cavity and not requiring a definitive diagnosis before surgery. Endometriosis can be diagnosed intraoperatively in some cases. For patients with multiple lesions, as in our case, surgery can be performed from the same port without losing these advantages and still be effective. Laparoscopic surgery for intestinal endometriosis is expected to become increasingly common in the future. However, to ensure that it is as safe as possible, the potential complications should be borne in mind. Endometriosis has a high postoperative recurrence rate, and postoperative hormone therapy and total hysterectomy/adnexectomy may eventually be necessary. Our patient became symptom-free, resumed infertility treatment soon after surgery, and had a successful term pregnancy. Laparoscopic surgery is minimally invasive but requires a skilled operator and advanced techniques. Adequate informed consent before surgery is essential.

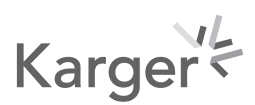


Nagakari et al.: Laparoscopic Bowel Resection for Endometriosis during Infertility Treatment

\section{Conclusion}

We have encountered a case of intestinal obstruction as a result of intestinal endometriosis that was detected during infertility treatment. Laparoscopic surgery was performed in three anatomic locations. Laparoscopic surgery may be useful for multiple endometriotic lesions and serve as a bridge to infertility treatment.

\section{Statement of Ethics}

This case report followed the principles of the Declaration of Helsinki and was approved by the Ethics Review Board of Juntendo University Urayasu Hospital (Approval No. 3-001). Written informed consent was obtained from the patient for publication of this case report and any accompanying images.

\section{Conflict of Interest Statement}

The authors have no conflicts of interest to declare.

\section{Funding Sources}

No external funding was utilized in the creation of this case report.

\section{Author Contributions}

K. Nagakari reviewed the literature, drafted the case report, and participated in preoperative and postoperative care. K. Nagakari and K. Azuma performed surgery on the patient. K. Takehara and M. Ohuchi reviewed the final manuscript. Y. Ishizaki and K. Sakamoto supervised the preparation of the manuscript. All authors were involved in interpretation of the imaging results and discussed the treatment plan. All authors approved the final version of the manuscript.

\section{Data Availability Statement}

All data generated or analyzed during this study are included in this article. Further inquiries can be directed to the corresponding author.

\section{References}

1 Macafee CHG, Greer HLH. Intestinal endometriosis. A report of 29 cases and survey of the literature. J Obstet Gynaec Br Comm. 1945 Aug;67:539-55.

2 Jubanvic KJ, Comite F. Extrapelvic endometriosis. Obstet Gynecol Clin North Am. 1997 Jun;24(2):411-40.

3 Remorgida V, Ferrero S, Fulcheri E, Ragni N, Martin DC. Bowel endometriosis: presentation, diagnosis, and treatment. Obstet Gynecol Surv. 2007 Jul;62(7):461-70.

4 Sampson JA. The development of the implantation theory for the origin of peritoneal endometriosis. Am J Obstet Gynecol. 1940 Oct;40(4):549-57.

5 Nezhat C, Li A, Falik R, Copeland D, Razavi G, Shakib A, et al. Bowel endometriosis: diagnosis and management. Am J Obstet Gynecol. 2018 Jun;218(6):549-62.

\section{Karger'}


Nagakari et al.: Laparoscopic Bowel Resection for Endometriosis during Infertility Treatment

6 Kim TD. Endometriosis of the Bowel. Dis Colon Rectum. 2020 Nov;63(11):1496-8.

7 Bazot M, Kermarrec E, Bendifallah S, Daraï E. MRI of intestinal endometriosis. Best Pract Res Clin Obstet Gynaecol. 2021 Mar;71:51-63.

8 Milone M, Mollo A, Musella M, Maietta P, Sosa Fernandez LM, Shatalova O, et al. Role of colonoscopy in the diagnostic work-up of bowel endometriosis. World J Gastroenterol. 2015 Apr 28;21(16):4997-5001.

9 Debus G, Schuhmacher I. Endometrial adenocarcinoma arising during estrogenic treatment 17 years after total abdominal hysterectomy and bilateral salpingo-oophorectomy: a case report. Acta Obstet Gynecol Scand. 2001 Jun;80(6):589-90.

10 Minelli L, Fanfani F, Fagotti A, Ruffo G, Ceccaroni M, Mereu L, et al. Laparoscopic colorectal resection for bowel endometriosis: feasibility, complications, and clinical outcome. Arch Surg. 2009 Mar;144(3):234-9.

11 Ruffo G, Stepniewska A, Crippa S, Serboli G, Zardini C, Steinkasserer M, et al. Laparoscopic ileocecal resection for bowel endometriosis. Surg Endosc. 2011 Apr;25(4):1257-62.

12 Cosma S, Ceccaroni M, Benedetto C. A pseudoneoplastic finding of deep endometriosis: laparoscopic triple segmental bowel resection. Wideochir Inne Tech Maloinwazyjne. 2014 Sep;9(3):463-7.

13 Darai E, Dubernard G, Coutant C, Frey C, Rouzier R, Ballester M. Randomized trial of laparoscopically assisted versus open colorectal resection for endometriosis: morbidity, symptoms, quality of life, and fertility. Ann Surg. 2010 Jun;251(6):1018-23.

14 Practice Committee of the American Society for Reproductive Medicine; Society of Reproductive Surgeons. Pathogenesis, consequences, and control of peritoneal adhesions in gynecologic surgery. Fertil Steril. 2007 Jul; 88(1):21-6. 